\title{
African swine fever in backyard pigs of Sabah state, East Malaysia, 2021
}

\author{
Khoo, C.K. ${ }^{1}$, Norlina, D. ${ }^{1}$, Roshaslinda, D. ${ }^{1}$, Siti Suraya Hani, M.S. ${ }^{1}$, Zunaida, B. ${ }^{1}$, Mohd Hasrul, A.H. ${ }^{1}$, \\ Pauzi, N.A.S. ${ }^{1}$, Roslina, H. ${ }^{1}$, Faizah Hanim, M.S. ${ }^{1}$, Leow, B.L. ${ }^{1 *}$
}

${ }^{1}$ Veterinary Research Institute, Ipoh, Perak, Malaysia

*Corresponding author: leowbl@hotmail.com; leow@dvs.gov.my

\section{ARTICLE HISTORY}

Received: 8 September 2021 Revised: 22 October 2021 Accepted: 24 October 2021 Published: 21 December 2021

\begin{abstract}
African swine fever (ASF) is a transboundary haemorrhagic viral disease that affected domestic and wild pigs of all ages. The disease is caused by African swine fever virus (ASFV) and was introduced to China in 2018 before spreading rapidly to neighbouring Asian countries. As such, putting countries free from ASF like Malaysia at risk. ASF is highly lethal with no vaccine or treatment available. In February 2021, we confirmed backyard pigs from various locations in Sabah were infected with ASF using real time polymerase chain reaction (realtime PCR). Further characterization of the Sabah ASFVs indicated that they were of p72 genotype II with intergenic region (IGR) variant II that displayed an addition tandem repeat sequence (TRS) insertion, similar to ASFV from Indonesia, Vietnam and China. These results indicate and support the transboundary expansion of a homogenotypic ASFV (p72 genotype II and IGR variant II) in the Europe and Asia-Pacific, emphasizing the need for a holistic international collaboration in control and preventing further spreading of the current ASF pandemic. Importantly, our results informed the first detection and characterization of ASF, a disease previously not detected in Malaysia. This information is crucial for further mitigation and preventive measures.
\end{abstract}

Keywords: African swine fever; Malaysia; p72; TRS; backyard pig.

\section{INTRODUCTION}

African swine fever virus (ASFV), the sole member of the Asfaviridae family, is the etiological agent for African swine fever (ASF). Both domestic and wild pigs (including warthogs, bush pigs, giant forest hogs, wild boars) of all ages are susceptible to ASF (Beltrán-Alcrudo et al., 2017). Nonetheless, the wild African suids are asymptomatic carrier and act as reservoirs of ASFV. There is neither vaccine nor treatment available for ASF (Dixon et al., 2020). Other than pigs, ASFV also infects ticks of the genus Ornithodoros, causing tickmediated ASF infection in warthogs (sylvatic cycle). Besides sylvatic cycle, ASFV can persists in tick-pig cycle, pig-pig cycle in both domestic and wild boar (Beltrán-Alcrudo et al., 2017). The disease transmission could be through direct contact with the infected live or dead pigs (domestic or wild) and indirect contact through contaminated pork products, feed and fomites including tick bite (Dixon et al., 2020).

ASF is a mandatory reportable disease in Malaysia and notifiable disease by the World Organisation for Animal Health (OIE). Although ASF is non-zoonotic and humans are not affected, the mortality rate in pigs is up to $100 \%$ (BeltránAlcrudo et al., 2017). The disease is highly contagious, characterized by a variety of clinical forms and often dominated by the hemorrhagic acute form (Blome et al., 2013). Moreover, ASFV has high environmental resistance. The virus is resilient and stable over a wide range of temperature, $\mathrm{pH}$ and resistant to autolysis and various disinfectants (BeltránAlcrudo et al., 2017; Kalmar et al., 2018). As such, ASFV shown remarkable abilities to transmit and spread rapidly, causing substantial socio-economic implications (Dixon et al., 2020). In Vietnam alone, nearly six million pigs were culled in the 2019 outbreak (GSO, 2021).

ASF is a re-emerging transboundary animal disease (TAD) of African origin. To date, most South-Saharan African countries, Sardinia, and parts of the Caucasus and Eastern Europe are considered endemic for ASF (Gallardo et al., 2014; Sanna et al., 2017). In the past three years, ASF has been introduced and rapidly expanded to large parts of the Asian region. In Asia- Pacific, ASF was first reported in Liaoning province in China in August 2018 (Ge et al., 2018) before spread rapidly throughout China. After incursion into China, ASF expanded rapidly into neighboring East Asia countries (Mongolia, Hong Kong, North Korea, South Korea), Southeast Asia countries (Vietnam, Cambodia, Laos, Philippines, Myanmar, Timor-Leste, Indonesia), Melanesia (Papua New Guinea) and India in South Asia (FAO, 2021).

ASFV is a complex double stranded DNA virus with genome size varied between 170 to $193 \mathrm{kbp}$ (Dixon et al., 2020). Depending on the virus strain, up to 150 to 167 protein coding open reading frames were reported on its genome. To date, genetic characterization of ASFV relied on sequence 
analysis of few different genetic regions (Sanna et al., 2017). The $B 646 \mathrm{~L}$ gene (encoding p72 major capsid protein) is a key fragment for ASFV genotyping (Bastos et al., 2003) with 24 geographically related genotypes reported so far (Ge et al., 2018). However, the abundant genotype of ASFV reflected the complexity of ASF epidemiology especially in discriminate closely related isolates. Therefore, variable tandem repeat sequences (TRS) between I73R and I329L gene was used as a tool for subtyping closely related ASFV from Eastern Europe, the current circulating strain in Europe and Asia region (Gallardo et al., 2014; Mazur-Panasiuk et al., 2020).

The ongoing uncontrolled spreads of ASF in the region have impacted the relevant industries hard and put countries free from ASF like Malaysia at risk. Unexpectedly, high sudden mortality in backyard pigs was reported in February 2021 at Sabah state, suspected for ASF. Here, we described the first detection along with the initial genetic characterization (partial p72 and TRS) of ASFV for the first outbreak in Malaysia.

\section{MATERIALS AND METHODS}

\section{Samples}

A total of 14 samples were received from the Sabah State Department of Veterinary Services (Sabah DVS) in February 2021 for the diagnosis of swine viral diseases including
ASF. The samples (bone marrow, liver, kidney, spleen, lung, intestine, heart and brain) were from cases of high sudden mortality involving backyard pigs in three neighboring districts (Pitas, Kota Marudu and Beluran) within Kudat and Sandakan division of Sabah, East Malaysia (Figure 1). The pigs were reported depressed and loss of appetite prior sudden death. The samples from different districts were received in different date and were processed separately according to standard operating protocol to avoid contamination.

Deoxyribonucleic acid (DNA) extraction and real time polymerase chain reaction (real-time PCR)

The specimens were homogenized with mortar and pestle in the presence of sterile sand. The homogenate was centrifuged at $3000 \mathrm{rpm}$ for 10 minutes before the supernatant was filtered. Viral DNA extraction was done from the supernatant with the IndiSpin Pathogen Kit (Indical Bioscience, Germany) according to the manufacturer's instructions. The extracted DNA was stored at $-20^{\circ} \mathrm{C}$ until further analysis. The ASFV detection was carried out by following the manufacturer's instructions of the real-time virotype ASFV PCR Kit (Indical Bioscience, Germany) which the endogenous control is included. The amplification was performed on CFX Connect Real-Time PCR System (Bio-Rad, USA). Besides that, primer-probe from King et al. (2003) as recommended by the

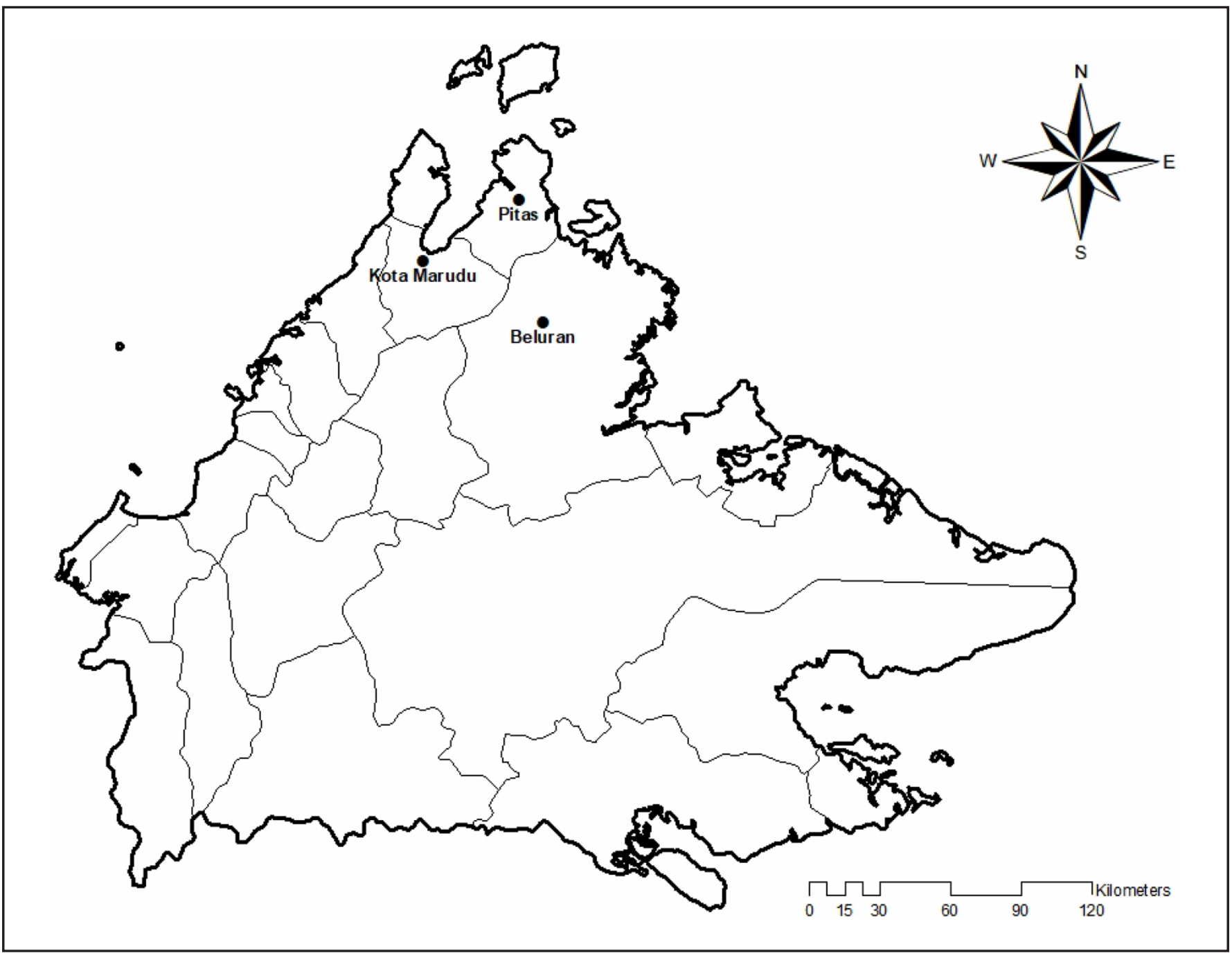

Figure 1. Map of Sabah State, East Malaysia shows the locations of ASF positive cases. The Sabah base map was used to map out the distribution of ASF cases according to World Geodetic System (WGS) 1984 projection with ArcGIS 10.8 by ESRI. 
OIE was also used to detect the ASFV (OIE, 2021a). For the King et al. (2003) probe, BHQ1 was used as quencher instead of TAMRA. The $15 \mathrm{uL}$ real-time PCR mixes contained final concentration of $0.5 \mathrm{pmol}$ of both sense and antisense primer, 0.5 pmol of probe and $1 \times$ LightCycler $^{\circledR}$ Taqman $^{\circledR}$ Master mix (Roche, Switzerland), before 5 uL of DNA was added. The reaction was subjected to $95^{\circ} \mathrm{C}$ for 10 minutes for initial denaturation, followed by 45 cycles of denaturation at $95^{\circ} \mathrm{C}$ for $10 \mathrm{sec}$, annealing at $60^{\circ} \mathrm{C}$ for $35 \mathrm{sec}$ and extension at $72^{\circ} \mathrm{C}$ for $1 \mathrm{sec}$ with QuantStudio 3 System (Applied Biosystems, USA).

\section{Gene sequencing and phylogenetic analysis}

For each district, one positive ASF sample was amplified and further characterized on the partial $B 646 L$ gene (encoding the p72 major capsid protein) (Bastos et al., 2003) and intergenic region (IGR) variant between I73R and I329L genes (Gallardo et al., 2014). Both of these molecular markers are used in the standard genotyping procedures (Ge et al., 2018; Le et al., 2019). The IGR between I73R and I329L genes contain tandem repeat sequence (TRS) that is important in distinguishing between closely related ASFVs. The conventional PCR was carried out with MyCycler ${ }^{\mathrm{TM}}$ (Bio-Rad, USA) using GoTaq $^{\circledR}$ Green Master Mixes (Promega, USA) in a total volume of $25 \mathrm{uL}$ reaction. The reactions were subjected to $95^{\circ} \mathrm{C}$ for 2 minutes for initial denaturation, followed by 35 cycles of denaturation at $95^{\circ} \mathrm{C}$ for $30 \mathrm{sec}$, annealing at $59^{\circ} \mathrm{C}$ for $30 \mathrm{sec}$, extension at $72^{\circ} \mathrm{C}$ for $30 \mathrm{sec}$ and a final extension at $72^{\circ} \mathrm{C}$ for 5 minutes. The amplicons were subjected to gel electrophoresis and visualized on $1.5 \%$ agarose gel, stained by SYBR ${ }^{\circledR}$ Safe DNA gel stain (Invitrogen, USA). The 100 bp DNA ladder (Promega, USA) was used as the molecular weight marker. The amplicons were then excised and sent for Sanger sequencing [Apical Scientifics (M) Sdn Bhd]. Raw nucleotide sequences were processed using BioEdit version 7.1.9 (Hall, 1999). The nucleotide sequences were deposited to GenBank and their accession numbers are MW788576-MW788578 (p72) and MW788579-MW788581 (TRS), respectively. Clustal W was used to align the partial p72 sequenced for Sabah ASFVs along with representative sequences for all 24 ASFV genotypes retrieved from GenBank. Phylogenetic analysis for partial p72 using neighbor-joining method with Kimura 2 parameter was run in 1000 bootstrap replicates with Molecular Evolutionary Genetics Analysis (MEGA) version 7 (Kumar et al., 2016).

\section{RESULTS}

All 14 samples (bone marrow, liver, kidney, spleen, lung, intestine, heart and brain) of backyard pigs from three different locations in Sabah were detected positive for ASFV by real-time PCR. In contrast, these samples were negative for others swine viral diseases including classical swine fever (CSF) and porcine reproductive and respiratory syndrome (PRRS). In addition, both virotype ASFV PCR Kit and King et al. (2003) assays detected consistent threshold cycle $\left(C_{t}\right)$ for all the samples. The $C_{t}$ value range from 18 to 27 with $\pm 0.5 C_{t}$ value difference between assays.

Further amplification and sequencing of one amplified product each from Pitas, Kota Marudu and Beluran with both p72 and TRS, confirmed and characterized the first occurrence and the outbreak of ASF in Malaysia. Analysis on the partial p72 shown all three Sabah strains were $100 \%$ identical to each other and to other ASFV strains from a wide geographical location, includes Indonesia, Vietnam and China. Phylogenetic analysis using partial p72 indicated the Sabah strain belonged to p72 genotype II (Figure 2). Analysis on the TRS revealed that all three Sabah ASFVs were IGR variant II with an additional $10 \mathrm{bp}$ TRS ( $5^{\prime}$-GGAATATATA-3') insertion between $173 R$ and $1329 L$ gene, compared to the reference sequence of the Georgia 2007 strain (Figure 3). These results were similar to ASFV strains from Indonesia, Vietnam and China.

\section{DISCUSSION}

Clinically, ASF is indistinguishable from other porcine diseases including CSF and PRRS, making differential diagnosis challenging. Moreover, the confirmation of ASF depends on laboratory testing (OIE, 2021a). In this study, both virotype ASFV PCR and King et al. (2003) assay showed specific detection and consistent performance among each other's. Together with the characterization markers, rapid reliable detection and confirmation of ASF are crucial for disease differential diagnosis and timely implementation of control measures in preventing disease spreading.

The p72 and IGR molecular markers have been used extensively in ASF phylogenetic and molecular epidemiological purposes. So far, there are 24 geographical related ASFV genotypes based on the p72 characterization (Ge et al., 2018). Notably, all three Sabah ASFVs were of p72 genotype II. Grouped in a monophyletic with ASFV strains from Indonesia, Vietnam, China, Estonia and Georgia, this ASFV strain is in accordance with the genotype that is responsible for the current ASF epizootic in Europe and Asia since its introduction into Georgia in 2007 (Ge et al., 2018; Le et al., 2019; Dharmayanti et al., 2021). In combination with the Asia and the Pacific ASF epidemiology, our result suggests potential spillover or introduction of this TAD from the neighboring countries concordance with the outbreak timeline.

Comparisons of TRS of Sabah strains with Indonesia, Vietnam, Korea, China, few other Eastern Europe countries and Georgia are also being carried out to explore the potential epidemiology and introduction of ASFV p72 genotype II into East Malaysia. Likewise, the Sabah strains show a homogenous nucleotide pattern with an additional 10 bp TRS (5'-GGAATATATA-3') insertion between I73R and I329L gene (Figure 3), which categorized them as an IGR variant II (Mazur-Panasiuk et al., 2020). This additional motif is absent in domestic pig isolate from Georgia, the older p72 genotype II isolate. Conversely, this motif is present in all recent ASFV strains isolated from domestic pigs from Asia and both domestic and wild pigs from Eastern Europe. Taken together, this information implied the current circulating strain may originate from the recent evolved strain compared to the original Georgia strain which was introduced from Africa in 2007 (Rowlands et al., 2008). Importantly, this information is in agreement with the current epidemiological events and reaffirmed the expansion of a homogenotypic ASFV from Europe to Asia-Pacific region.

All the Sabah strains were identical to each other albeit been sampled from distant locations. The three districts (Pitas, Kota Marudu and Beluran) in this study were separated by the Crocker Range with the shortest road access connecting Kota Marudu to Beluran via Pitas. The distance between each location ranges from approximately $66 \mathrm{~km}$ to over $150 \mathrm{~km}$ through rough terrain. This emphasized the Sabah ASFVs are from the same introduction and indicated the disease had spread. To date, a total of 33 outbreaks have been officially confirmed across Sabah, involving both domestic pigs and wild boars with over 1100 domestic pigs have died or killed (OIE, 2021b). 


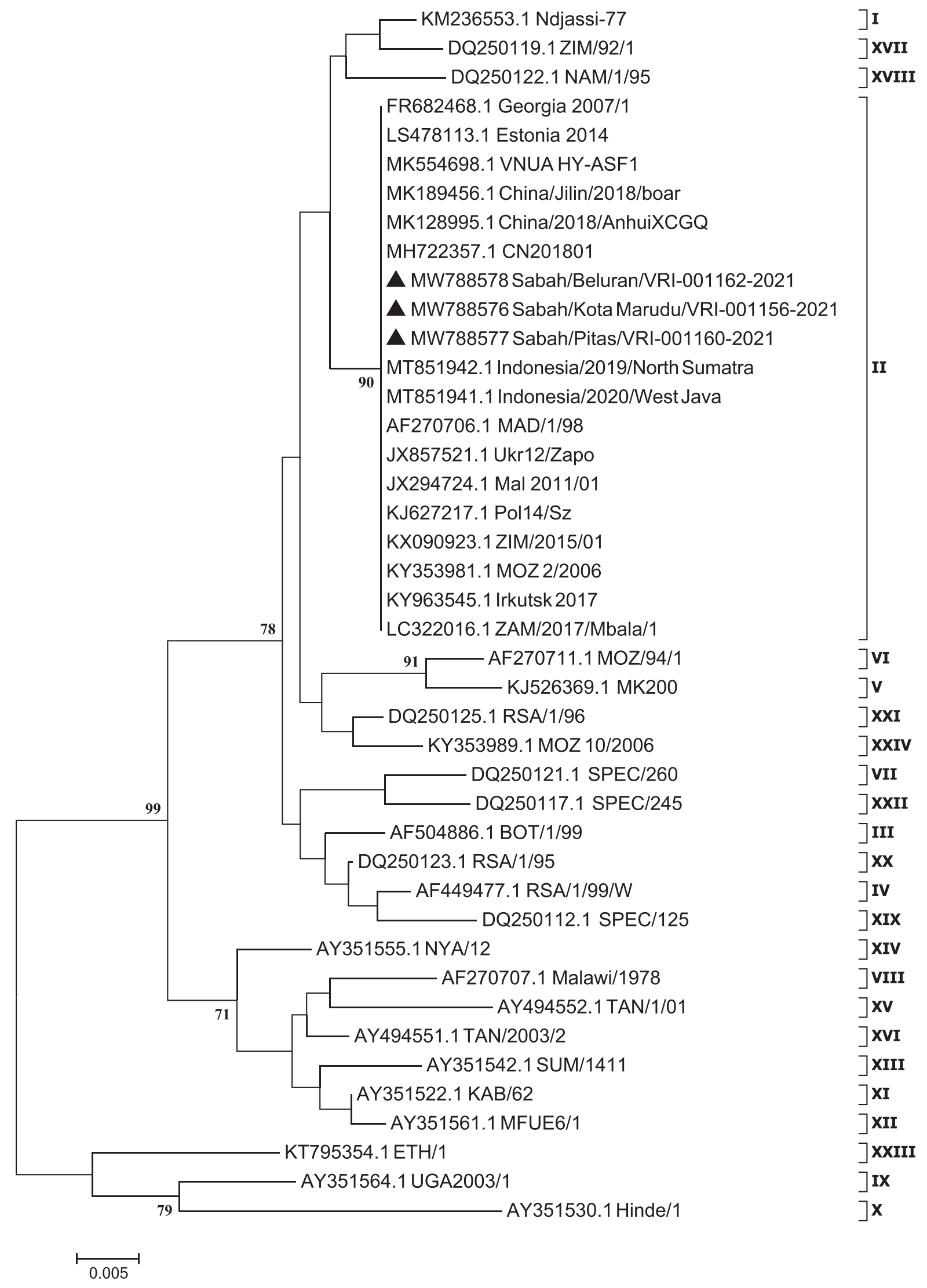

Figure 2. Phylogenetic analysis of the partial p72 gene of Sabah ASFV strains. The evolutionary history was inferred with the Neighbor-Joining method using Kimura 2 parameter model in MEGA 7 at 1000 bootstrap replicates. Only bootstrap values $>70 \%$ are shown along the branches. Black triangle indicates causative virus from this study. Roman numerals to the right indicate $\mathrm{p} 72$ genotypes. GenBank accession numbers are provided for all sequences. Scale bar indicates nucleotide substitutions per site. 


\title{
Sabah/Pitas/VRI-001160-2021
}

\author{
$90 \quad 100$ \\ 110

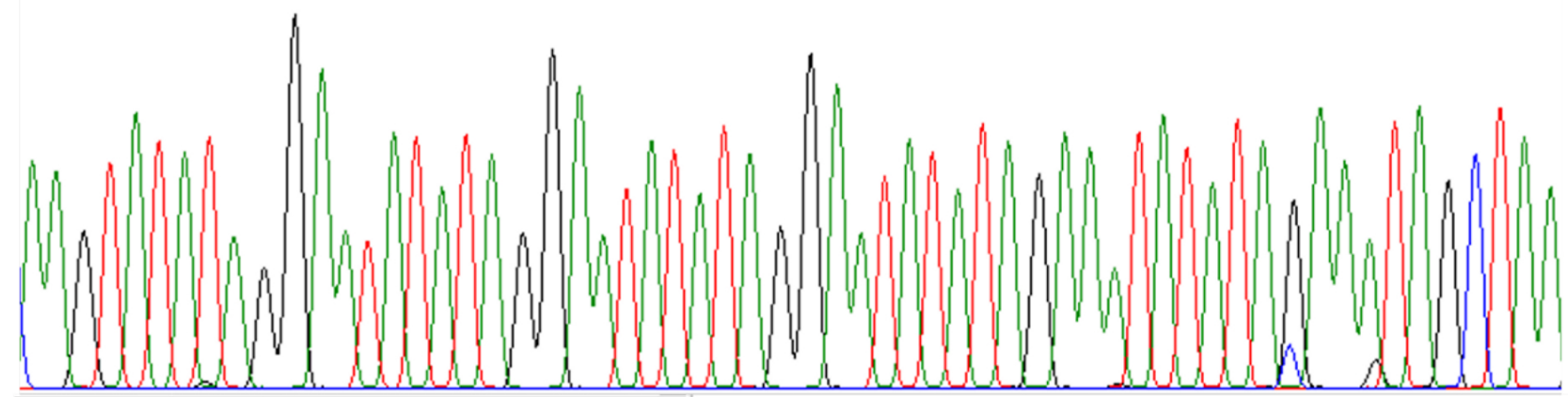

FR682468.2_Georgia/2007/1

RJ620037.1-Ukraine 12/Zapo

AagtatatagGatatataggatatata

GaAatatatagaAatagctaA

KJ620043.1_Belarus 13/Grodno

RJ620045.1_Lithuania 14/1482

KJ620046.1_Poland 14/Sz

MH735144.1_China/CN201801

MN603969.1_Korea/Pig/Paju1/2019

MN733934.1_Vietnam/Pig/HT/1205

MT851948.1 Indonesia/2019/Pig/North Sumatra

MT851947.1 Indonesia/2020/Pig/West Java

MW788579 Sabah/Rota Marudu/VRI-001156-2021

MW788580 Sabah/Pitas/VRI-001160-2021

MW788581 Sabah/Beluran/VRI-001162-2021

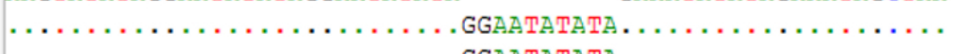

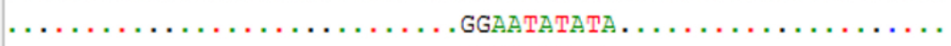

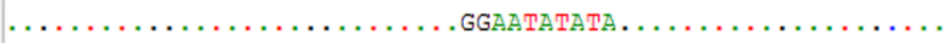

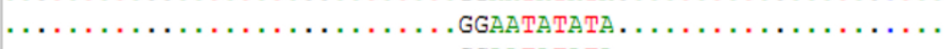

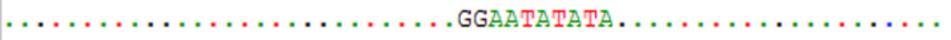

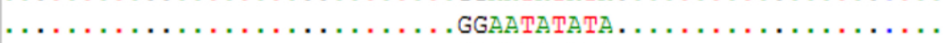

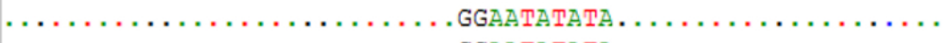

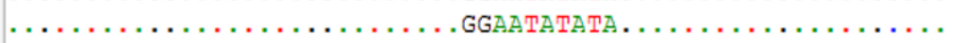

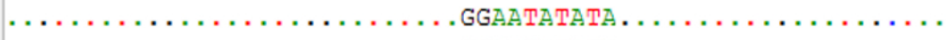

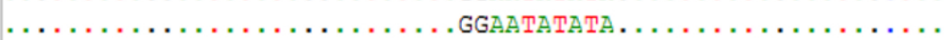

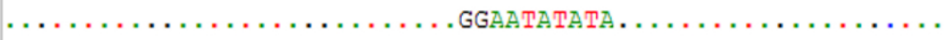

. . . . . . . . . . . . . . . . . . . . . . . . . . .

Figure 3. Analysis of the IGR variant between I73R and I329L gene of ASFV. The chromatogram shows the presence of three GGAATATATA motifs as highlighted in red colored rectangular in one of the Sabah isolates. Multiple sequence alignment of the IGR of Sabah ASFVs with other genotype II viruses from Asia, Eastern and Central Europe shows additional insertion of GGAATATATA motif in all isolates except isolate from Georgia. The dots show the conserved nucleotides. The dashes show absence of the additional GGAATATATA motif.

There are needs for further characterization of the Sabah ASFV especially on the wild boars sample which is lacking, to establishing the source of disease introduction and spread (Vergne et al., 2020). Notably, whole genome sequencing of ASFV could shed light on better understanding of the epidemiology, evolution and useful in distinguish the circulating strains on the genome scale. Collectively, this study provides evidence and supports the common origin and transboundary expansion of a homogenotypic ( $p 72$ genotype II and IGR variant II) circulating ASFV strain in AsiaPacific region.

The rapid transboundary transmission of this homogenotypic ASFV strain could be from a variety ways (Dixon et al., 2020; Vergne et al., 2020), particularly through infected pork products, swill feeding and loose border vigilance. However, the introduction of ASF into Sabah, East Malaysia remains to be elucidated. At present, there is no vaccine available for ASF. As such, the best strategies for infected countries like Malaysia are the implementation of stamping out, cleaning and disinfection, zoning and movement control, strengthen awareness, strict biosecurity, surveillance and rapid disease detection to contain and control the disease spreading.

The rapid transboundary expansion of ASF in this region is unprecedented. As such, holistic international collaborations encompass One Health paradigms are needed for timely response, effective implementation of control measures to mitigate the further spreading of ASF pandemic in this region and beyond.

\section{ACKNOWLEDGMENTS}

We thank the Sabah DVS for providing the samples, Mohammad Masrin Azami for help in plotting the map, The Pirbright Institute for providing the positive ASFV DNA. We would like to thank the Director General of Veterinary Services Malaysia and Director of Veterinary Research Division for their kind permission to publish the paper. Financial support for this research was provided by the Veterinary Research Institute, Ipoh Malaysia.

\section{Conflict of interest}

The authors declare that they have no conflict of interest.

\section{REFERENCES}

Bastos, A.D., Penrith, M.L., Crucière, C., Edrich, J.L., Hutchings, G., Roger, F., Couacy-Hymann, E. \& Thomson, G.R. (2003). Genotyping field strains of African swine fever virus by partial p72 gene characterisation. Archives of Virology 148: 693-706. https://doi.org/10.1007/s00705-002-0946-8

Beltrán-Alcrudo, D., Arias, M., Gallardo, C., Kramer, S. \& Penrith, M.L. (2017). African swine fever: detection and diagnosis - A manual for veterinarians. FAO Animal Production and Health Manual No. 19. Rome. Food and Agriculture Organization of the United Nations (FAO), pp. 88.

Blome, S., Gabriel, C. \& Beer, M. (2013). Pathogenesis of African swine fever in domestic pigs and European wild boar. Virus Research 173: 122-130. https://doi.org/10.1016/ j.virusres.2012.10.026 
Dharmayanti, N.I., Sendow, I., Ratnawati, A., Settypalli, T., Saepulloh, M., Dundon, W.G., Nuradji, H., Naletoski, I., Cattoli, G. \& Lamien, C.E. (2021). African swine fever in North Sumatra and West Java provinces in 2019 and 2020, Indonesia. Transboundary and Emerging Diseases 68: 28902896. https://doi.org/10.1111/tbed.14070

Dixon, L.K., Stahl, K., Jori, F., Vial, L. \& Pfeiffer, D.U. (2020). African swine fever epidemiology and control. Annual Review of Animal Biosciences 8: 221-246. https://doi.org/ 10.1146/annurev-animal-021419-083741

Food and Agriculture Organization of the United Nations (FAO). (2021). ASF situation in Asia update. https://www. fao.org/ag/againfo/programmes/en/empres/ASF/ situation_update.html. Accessed 19 February 2021.

Gallardo, C., Fernández-Pinero, J., Pelayo, V., Gazaev, I., Markowska-Daniel, I., Pridotkas, G., Nieto, R., FernándezPacheco, P., Bokhan, S., Nevolko, O. et al. (2014). Genetic variation among African swine fever genotype II viruses, eastern and central Europe. Emerging Infectious Diseases 20: 1544-1547. https://doi.org/10.3201/eid2009.140554

Ge, S., Li, J., Fan, X., Liu, F., Li, L., Wang, Q., Ren, W., Bao, J., Liu, C., Wang, H. et al. (2018). Molecular characterization of African swine fever virus, China, 2018. Emerging Infectious Diseases 24: 2131-2133. https://doi.org/10.3201/eid2411. 181274

General Statistics Office of Vietnam (GSO) (2021). Livestock of pig has recovered. https://www.gso.gov.vn/en/dataand-statistics/2021/04/livestock-of-pig-has-recovered/ Accessed 1 September 2021.

Hall, T.A. (1999). BioEdit: A user-friendly biological sequence alignment editor and analysis program for Window 95/ 98/NT. Nucleic Acids Symposium Series 41: 95-98.

Kalmar, I.D., Cay, A.B. \& Tignon, M. (2018). Sensitivity of African swine fever virus (ASFV) to heat, alkalinity and peroxide treatment in presence or absence of porcine plasma. Veterinary Microbiology 219: 144-149. https://doi.org/10.1016/ j.vetmic.2018.04.025

King, D.P., Reid, S.M., Hutchings, G.H., Grierson, S.S., Wilkinson, P.J., Dixon, L.K., Bastos, A.D. \& Drew, T.W. (2003). Development of a TaqMan PCR assay with internal amplification control for the detection of African swine fever virus. Journal of Virological Methods 107: 53-61. https://doi.org/ 10.1016/s0166-0934(02)00189-1
Kumar, S., Stecher, G. \& Tamura, K. (2016). MEGA7: Molecular Evolutionary Genetics Analysis version 7.0 for bigger datasets. Molecular Biology and Evolution 33: 1870-1874. https://doi.org/10.1093/molbev/msw054

Le, V.P., Jeong, D.G., Yoon, S.W., Kwon, H.M., Trinh, T., Nguyen, T.L., Bui, T., Oh, J., Kim, J.B., Cheong, K.M. et al. (2019). Outbreak of African swine fever, Vietnam, 2019. Emerging Infectious Diseases 25: 1433-1435. https://doi.org/10.3201/ eid2507.190303

Mazur-Panasiuk, N., Walczak, M., Juszkiewicz, M. \& Woźniakowski, G. (2020). The spillover of African swine fever in western Poland revealed its estimated origin on the basis of 0174L, K145R, MGF 505-5R and IGR I73R/I329L genomic sequences. Viruses 12: 1094. https://doi.org/10. 3390/v12101094

Rowlands, R.J., Michaud, V., Heath, L., Hutchings, G., Oura, C., Vosloo, W., Dwarka, R., Onashvili, T., Albina, E. \& Dixon, L.K. (2008). African swine fever virus isolate, Georgia, 2007. Emerging Infectious Diseases 14: 1870-1874. https://doi.org/ 10.3201/eid1412.080591

Sanna, G., Dei Giudici, S., Bacciu, D., Angioi, P.P., Giammarioli, M., De Mia, G.M. \& Oggiano, A. (2017). Improved strategy for molecular characterization of African swine fever viruses from Sardinia, based on analysis of p30, CD2V and $173 \mathrm{R} / \mathrm{I329L}$ variable regions. Transboundary and Emerging Diseases 64: 1280-1286. https://doi.org/10.1111/ tbed.12504

Vergne, T., Guinat, C. \& Pfeiffer, D.U. (2020). Undetected circulation of African swine fever in wild boar, Asia. Emerging Infectious Diseases 26: 2480-2482. https://doi.org/ 10.3201/eid2610.200608

World Organisation for Animal Health (OIE). (2021a). OIE Terrestrial Manual. Chapter 3.9.1. African swine fever (infection with African swine fever virus). https://www. oie.int/fileadmin/Home/eng/Health_standards/tahm/ 3.09.01_ASF.pdf. Accessed 28 August 2021.

World Organisation for Animal Health (OIE). (2021b). OIEWAHIS. African swine fever virus (inf. with), Malaysia. https://wahis.oie.int/\#/report-info?reportld=35959. Accessed 28 August 2021. 